\title{
Broadband Flexible Fully Textile-Integrated Bandstop Frequency Selective Surface
}

\author{
Leticia Alonso-González, Student Member, IEEE, Samuel Ver-Hoeye, Member, IEEE, \\ Miguel Fernández-García, and Fernando Las-Heras Andrés, Senior Member, IEEE
}

\begin{abstract}
A broadband flexible fully textile-integrated bandstop frequency selective surface working at a central frequency of $5 \mathrm{GHz}$ and presenting a $1.8 \mathrm{GHz}$ bandwidth has been designed, manufactured and experimentally characterised. The frequency selective surface consists of two isolated layers of periodic square-shaped conductive rings and, due to its symmetries, its performance is largely independent of polarisation and angle of incidence. The textile frequency selective surface has been simulated and experimentally validated under bent conditions providing a stable performance.
\end{abstract}

Index Terms-Flexible, frequency selective surface (FSS), bandstop, textile, textile-integrated circuits.

\section{INTRODUCTION}

D URING the last few years, there has been an overwhelming interest in frequency selective surfaces (FSS) due to the wide number of applications in which they can be used [1], such as absorbers [2], artificial magnetic conductors [3], electromagnetic shielding, among others. The electromagnetic shielding can be achieved thanks to the bandpass [4] or bandstop [5, 6] responses of the FSS, depending on the periodic structure.

Different approaches have been used to develop FSS, from conventional designs based on resonators implemented over rigid substrates [7-9], FSS based on 3D printing [10], the use of high permittivity ceramic materials in order to avoid conductive materials [11], thicker multiband designs based on substrate integrated waveguide (SIW) technology [12] or multilayered designs [13]. The before mentioned alternatives present different advantages, such as the possibility of achieving high precision details in the fabricated prototypes. Nevertheless, none of them are flexible or washable through industrial processes, and their dimensions are constrained by the prototyping machine or $3 \mathrm{D}$ printer, leading to a limited number of unit cells in the manufactured design.

Some solutions have also been developed to integrate the FSS into the textile. As an example, in [14], a flexible and portable textile-reflectarray backed by an FSS is proposed using two separate embroidered fabrics connected through Velcros. However, this solution requires two fabrics and is not appropiate for low profile applications. In [15], an inkjet

This work has been supported by Gobierno de España TEC2015-72110EXP, MINECO-17-TEC2016-80815-P and FPU14/00016 grant, and by the Gobierno del Principado de Asturias (PCTI)/FEDER-FSE under projects IDI/2016/000372 and IDI/2017/000083.

The authors are with the Signal Theory and Communications Area, Department of Electrical Engineering, University of Oviedo, Gijón E-33203, Spain (e-mail: alonsoleticia@uniovi.es - lalonso@tsc.uniovi.es). printed FSS over a fabric is presented, whereas in [16], an FSS is proposed employing the screen printing technology. These procedures present the difficulty of achieving a thin and conductive printed layer over an uneven substrate, leading to the necessity of double-printing the layout or using intermediate impermeable coatings between the fabric and the ink, increasing the number of required subprocesses. Furthermore, the ink must be properly printed on the fabric to avoid its deterioration due to the wear out.

With the aim of pushing textile components to the microwave range, a weaving-based procedure to design, simulate and manufacture RF textile-integrated structures has already been thoroughly discussed in $[17,18]$. Moreover, this weavingbased procedure presents the advantage of avoiding posterior adhesives, coatings or sewing procedures to manufacture the flexible prototypes. For applications such as momentarily shielding a room from a certain frequency, a flexible woven structure can work as a smart curtain, which is easy to install, uninstall, keep or transport. Moreover, industrial weaving looms allow the automatically manufacturing of very large prototypes, in comparison with standard prototyping machines. For this reason, flexible textile-integrated FSS can overcome the problems attributed to standard FSS, opening a new field of research.

In this paper, a broadband flexible fully textile-integrated bandstop FSS has been designed. It can be manufactured with existing industrial textile machinery, making it suitable for mass production in larger dimensions than conventional FSS based on rigid substrates. The proposed FSS is based on two isolated layers of periodic conductive square-rings and its performance is independent of the incident wave direction and polarisation. Moreover, the proposed FSS has been simulated and experimentally validated under bent conditions, providing a stable performance.

The paper is organised as follows. In Section II, the structure of the FSS will be explained. In Section III, a description of the employed materials will be presented. In Section IV, the translation into a woven FSS will be discussed. In Section V, the simulated data will be presented. In Section VI, the fabrication process of the FSS will be explained. In Section VII, the experimental validation will be presented and discussed.

\section{Structure OF THE FSS}

A broadband bandstop FSS is proposed to work at $5 \mathrm{GHz}$ with a $1.8 \mathrm{GHz}$ bandwidth. The FSS has been designed from a unit cell to which periodic boundary conditions (PBC) 
TABLE I

DIMENSIONS OF THE FSS

\begin{tabular}{cccccc}
\hline \multicolumn{5}{c}{ Dimensions $(\mathrm{mm})$} \\
\hline \hline \multicolumn{2}{c}{ Top layer } & \multicolumn{2}{c}{ Bottom layer } & \multicolumn{2}{c}{ Unit cell } \\
\hline$L_{\mathrm{T}}$ & $W_{\mathrm{T}}$ & $L_{\mathrm{B}}$ & $W_{\mathrm{B}}$ & $L$ & $H$ \\
34 & 5 & 30 & 7.5 & 45 & 1 \\
\hline
\end{tabular}

have been applied, leading to an ideal infinite FSS. Figure 1a represents an overview of the unit cell and the boundary conditions. The tangential component of the magnetic field, $H_{\mathrm{T}}$, in the two boundaries which are parallel to the YZ-plane is zero and the tangential component of the electric field, $E_{\mathrm{T}}$, in the two boundaries which are parallel to the XZ-plane is zero, leading to a PBC environment. The unit cell has been iluminated with a plane wave, whose polarisation axis is vertically oriented (Y-axis), propagating in the $\hat{\mathrm{k}}$ direction from port 1 to port 2 , so that the $S_{21}$ parameter can be calculated.

Figure $1 \mathrm{~b}$ depicts a side view of the unit cell and its layers. The unit cell is composed of three layers, the top and the bottom layers which are separated by an isulating layer (middle layer), leading to a total height of the unit cell denoted by $H$. The top layer of the unit cell is composed of a square-shaped ring resonator, whose side and width are $L_{\mathrm{T}}$ and $W_{\mathrm{T}}$, respectively, as it can be seen in Fig. 1c. Similarly, the bottom layer of the unit cell is composed of a squareshaped ring resonator, whose side and width are $L_{\mathrm{B}}$ and $W_{\mathrm{B}}$, respectively, as it can be seen in Fig. 1d. The total length of the square-shaped unit cell is $L$. All the mentioned dimensions are summarised in Table I.

The proposed FSS is fully integrated in textile, therefore, the conductive and dielectric parts are manufactured using different types of threads. The employed materials will be explained in Section III and the translation into a woven prototype will be detailed in Section IV. For this reason, the dimensions summarised in Table I have been calculated once a sample of the textile substrate has been electromagnetically characterised, as it will be explained in Section IV.

\section{DESCRIPTION OF THE EMPlOYED MATERIALS}

Different materials have been employed for the design of the FSS, for both the weft and the warp directions. The weft direction coincides with the width of the fabric and, consequently, with the width of the loom, while the warp direction coincides with the length of the fabric. The warp threads are previously assembled in the loom, whereas the weft yarns are successively inserted in the woven structure during the fabrication process. In the proposed FSS, the warp direction coincides with the direction of the Y-axis. Consequently, the weft direction is oriented in the $\mathrm{X}$-axis direction. In this section, a description of the employed materials and, therefore, the structure of the woven substrate is presented.

\section{A. Description of the Electrically Conductive Materials}

Electrically conductive Shieldex 117f17 2-ply yarns, [19], with a resistance of $3.9 \Omega / \mathrm{cm}$ have been used for the conduc-

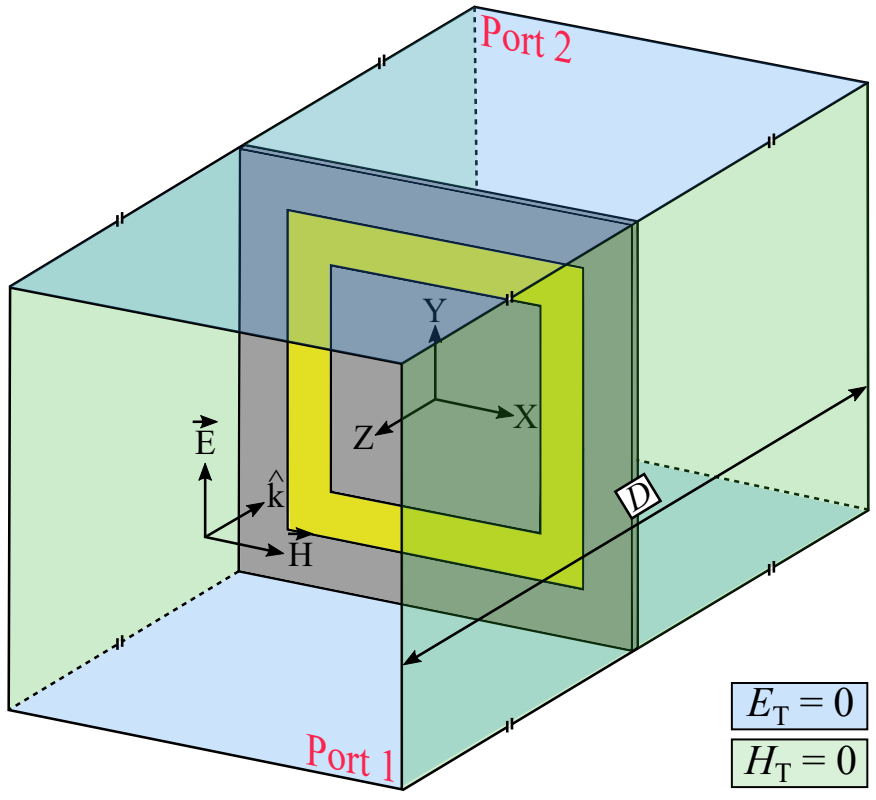

(a)

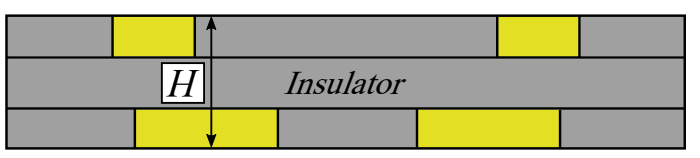

Top layer Middle layer Bottom layer

(b)

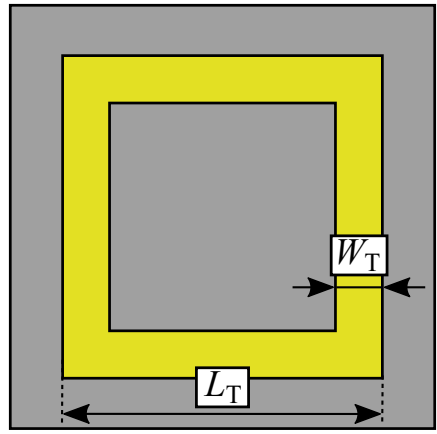

(c)

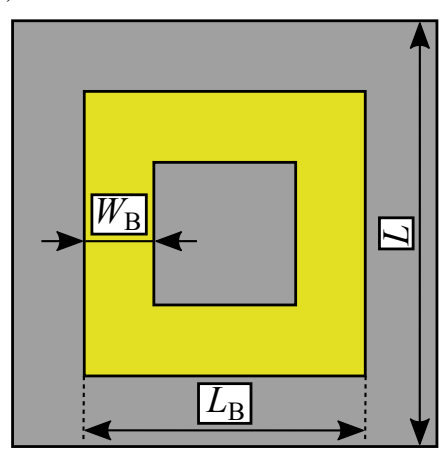

(d)
Fig. 1. Schematic drawing of the proposed unit cell, conductive materials (yellow) and dielectric materials (grey). The dimensions are summarised in Table I. (a) Overview of the unit cell and boundary conditions $(D=1.5 \mathrm{~m})$. (b) Side view of the unit cell and its layers. (c) Top layer of the unit cell and its dimensions. (d) Bottom layer of the unit cell and its dimensions.

tive warp and weft threads. The ply number means the number of initial threads that are twisted around one another to create a single and stronger thread. These threads consist of two plies and each ply is formed by 17 filaments and weights 117 dtex. The diameter of the Shieldex $117 f 17$ 2-ply yarns is $d_{\text {Shieldex }}=$ $0.3 \mathrm{~mm}$. Figure 2a shows a sample of a Shieldex $117 f 17$ 2-ply yarn, whereas in Fig. $2 b$ the unraveled filaments from a single ply are depicted.

\section{B. Description of the Dielectric Materials}

For the dielectric parts of the FSS two different types of threads have been used. For the weft threads, high tenacity white polyethersulfone (commonly known as PES) 550 dtex has been employed. The diameter of the PES yarns is $d_{\mathrm{PES}}$ 


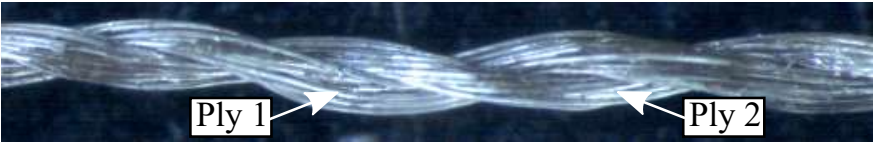

(a)

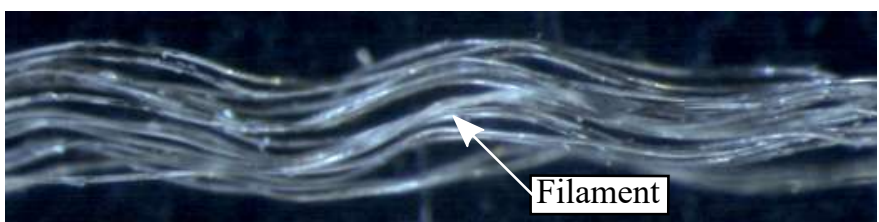

(b)

Fig. 2. Employed conductive thread. (a) Shieldex $117 \mathrm{f} 17$ 2-ply thread sample. (b) Unraveled filaments from one single ply.

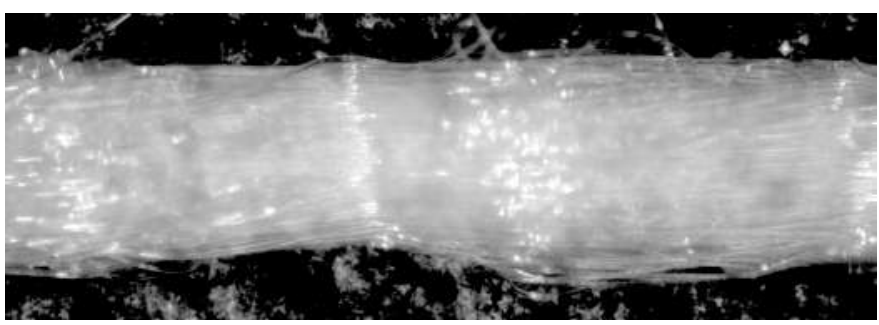

(a)

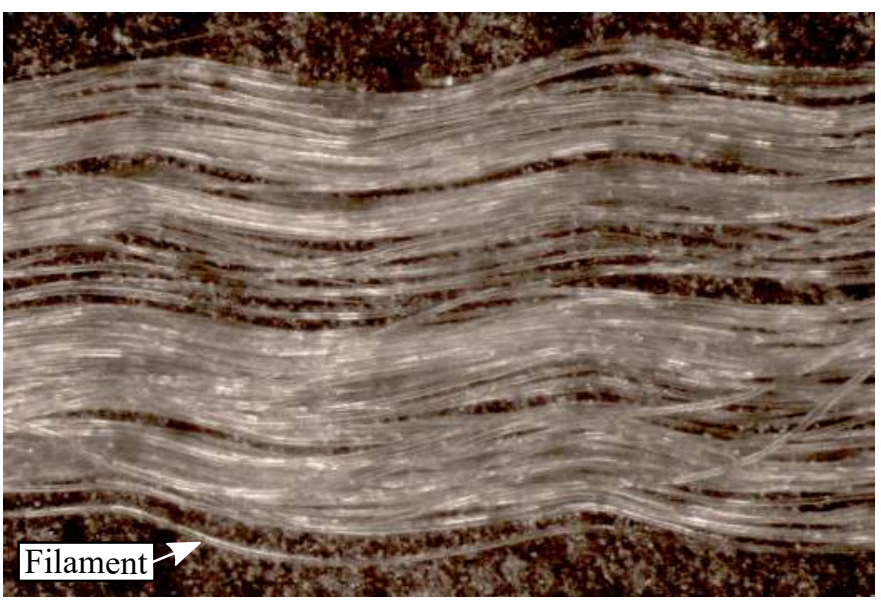

(b)

Fig. 3. Employed dielectric weft thread. (a) High tenacity PES 550 dtex thread sample. (b) Unraveled filaments.

$=0.3 \mathrm{~mm}$. Figure $3 \mathrm{a}$ shows a sample of a high tenacity PES thread, whereas Fig. $3 \mathrm{~b}$ describes the unraveled filaments of the sample sample. For the warp threads, white polyethyleneterephthalate (commonly known as PET or polyester) $76 f 24$, which means that it is composed of 76 filaments with 24 dtex, has been used. The diameter of the PET yarns is $d_{\mathrm{PET}}=$ $0.1 \mathrm{~mm}$. Figure $4 \mathrm{a}$ depicts a sample of a PET $76 f 24$ thread, whereas Fig. $4 \mathrm{~b}$ shows the unraveled filaments of the same sample.

\section{Design of The Woven FSS}

A broadband bandstop FSS is proposed. The FSS has been designed from a unit cell to which PBC has been applied, leading to an ideal infinite FSS. Nevertheless, a finite version composed of $9 \times 9$ unit cells has also been simulated

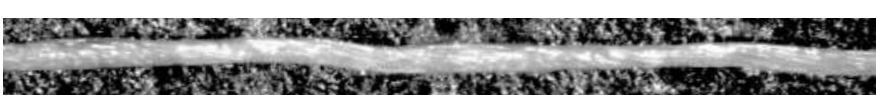

(a)

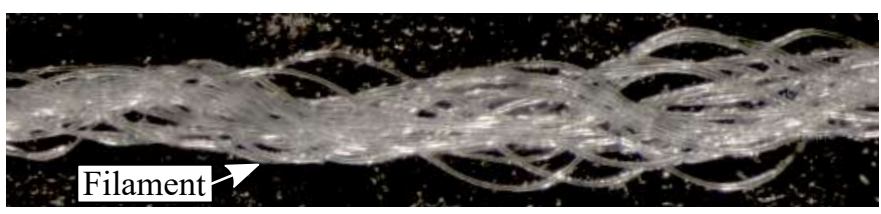

(b)

Fig. 4. Employed dielectric warp thread. (a) PET 76f 24 dtex thread sample. (b) Unraveled filaments.

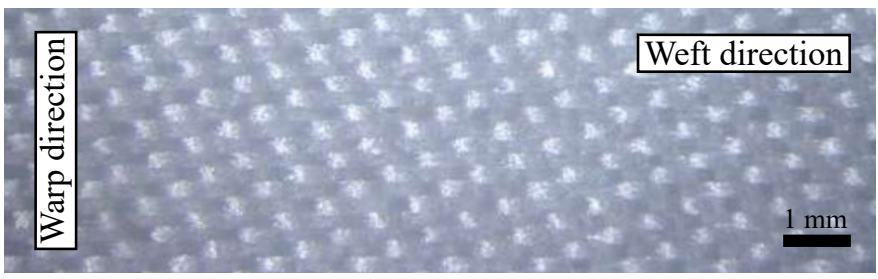

(a)

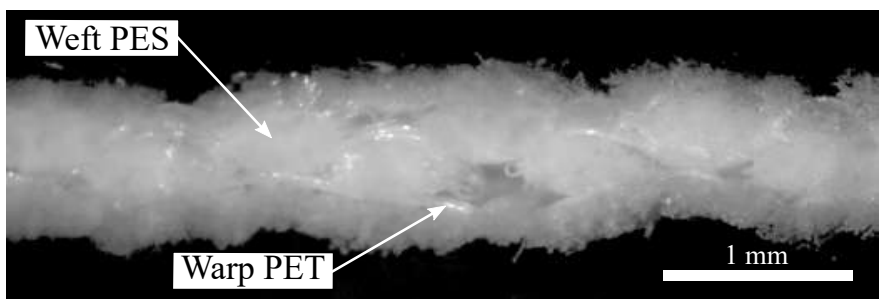

(b)

Fig. 5. Woven substrate. (a) Top view. (b) Side view.

to compare the simulations with the measurements. First, a sample of the dielectric substrate has been manufactured and electromagnetically characterised in order to design the FSS and, therefore, translate it into a woven prototype.

The substrate of the FSS is a multilayered fabric composed of three layers of weft PES threads interwoven using warp PET yarns, leading to a satin woven structure. Due to the air gaps between the threads, a sample of the substrate has been manufactured and electromagnetically characterised using an Agilent Technologies 85072A Split Cylinder Resonator and its relative dielectric permittivity and loss tangent have been found to be $\varepsilon_{\text {subs }}=1.7$ and $\tan (\delta)_{\text {subs }}=0.00362$, respectively. Figure 5a represents the top view of a sample of the woven substrate whereas Fig. 5b shows a side view of the woven substrate.

The woven prototype is composed of three layers: two dielectric layers in which the conductive square-rings are inserted, separated by an insulating layer. The three layers are identical, except for the positions where the dielectric threads have been substituted by conductive threads to implement the resonators. The conductive square rings placed in the top layer are translated into 5 Shieldex threads as shown in Fig. $6 a$, which will be simulated as 5 conductive strips, separated a distance $s_{\mathrm{T}}$ between centres and whose widths are $d_{\mathrm{T}}=$ $d_{\text {Shieldex }}$, as it can be seen in Fig. 6c. The conductive square rings placed in the bottom layer are translated into 7 Shieldex 


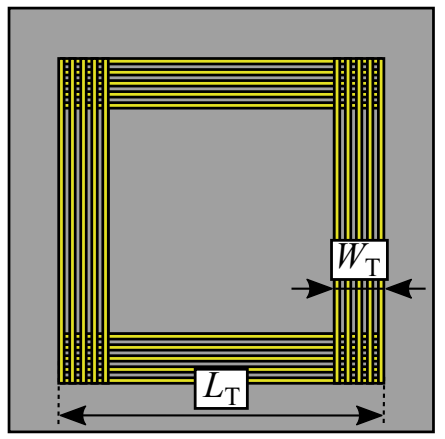

(a)

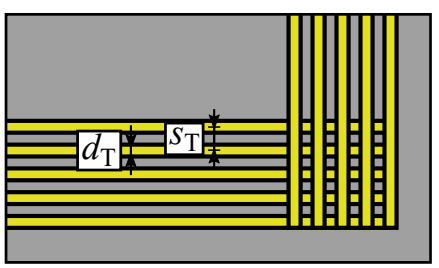

(c)

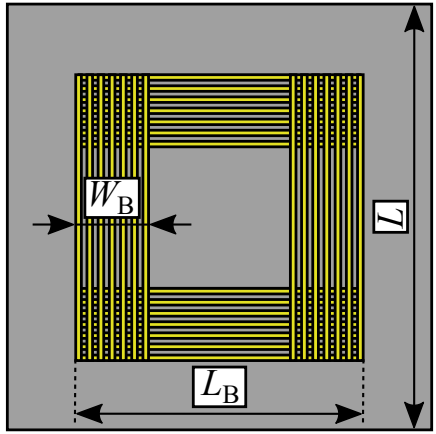

(b)

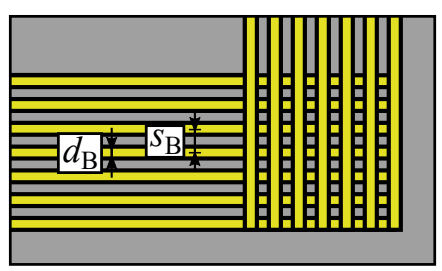

(d)
Fig. 6. Schematic drawing of the proposed unit cell and its dimensions The dimensions are summarised in Table II. (a) Top view. (b) Bottom view. (c) Detailed view of the dimensions (top view). (d) Detailed view of the dimensions (bottom view).

TABLE II

DIMENSIONS OF THE WOVEN FSS

\begin{tabular}{cccccccccc}
\hline \multicolumn{10}{c}{ Dimensions $(\mathrm{mm})$} \\
\hline \hline \multicolumn{10}{c}{ Top layer } \\
\hline$L_{\mathrm{T}}$ & $W_{\mathrm{T}}$ & $d_{\mathrm{T}}$ & $s_{\mathrm{T}}$ & $L_{\mathrm{B}}$ & $W_{\mathrm{B}}$ & $d_{\mathrm{B}}$ & $s_{\mathrm{B}}$ & $L$ & $H$ \\
34 & 5 & 0.3 & 1.175 & 30 & 7.5 & 0.3 & 1.2 & 45 & 1 \\
\hline
\end{tabular}

threads as shown in Fig. 6b, which will be simulated as 7 conductive strips, separated a distance $s_{\mathrm{B}}$ between centres and whose widths are $d_{\mathrm{B}}=d_{\text {Shieldex }}$, as it can be seen in Fig. $6 \mathrm{~d}$. The mentioned dimensions are summarised in Table II.

\section{Simulations}

The proposed design has been analysed using the 3D fullwave High Frequency Structure Simulator (HFSS) software. First, an infinite FSS modelled as one unit cell (described in Section IV) with PBC has been simulated under normal incidence conditions and the result has been compared with the simulation of a finite structure. Then, the behaviour of the finite FSS in terms of angle of polarisation, angle of incidence and radius of curvature has been studied.

Two infinite arrays of resonators have been simulated applying $\mathrm{PBC}$ to a unit cell placing two ports at a distance of $D / 2=0.75 \mathrm{~m}$ from each side of the FSS, respectively, as previously illustrated in Fig. 1a. In Fig. 7, the individual behaviour of the resonators, using PCB, in the top and bottom layers has been represented. The resonators in the top and bottom layers present a bandstop behaviour at a central frequency of $3.4 \mathrm{GHz}$ and $5.1 \mathrm{GHz}$, respectively. The simulated individual behaviour of the electric field is depicted in Fig. 8. Figure 8a represents the electric field at $5.1 \mathrm{GHz}$ for a unit cell with PBC composed only of the top layer resonators, whereas Fig. 8b represents

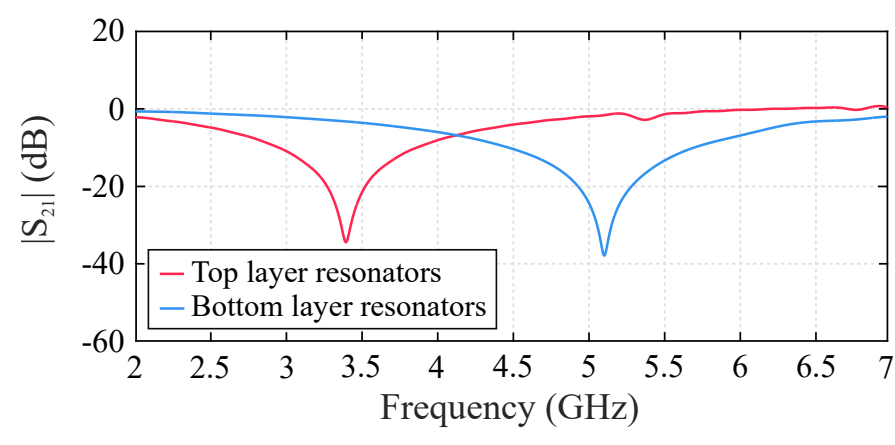

Fig. 7. Simulated individual behaviour of the resonators in the top and bottom layers using the PCB conditions.

their behaviour at $3.4 \mathrm{GHz}$. Figure $8 \mathrm{c}$ represents the electric field at $3.4 \mathrm{GHz}$ for a unit cell with $\mathrm{PBC}$ composed only of the bottom layer resonators, whereas Fig. 8d represents their behaviour at $5.1 \mathrm{GHz}$.

An infinite FSS (using both arrays of resonators) has been simulated, leading to a broadband bandstop behaviour of the complete FSS, as a result of the coupling phenomena between the two layers of resonators. A finite version $9 \times 9$ unitcell has also been simulated in the middle between two horn antennas separated a distance $D$. The simulated $S_{21}$ parameter, normalised by the response in the abscence of the FSS, is shown in Fig. 9a. The dimensions of the simulated horns are identical to the dimensions of the real horns which have been used for the experimental validation. The comparison between the simulation of both $\left|S_{21}\right|$ parameters can be seen in Fig. 10. The non-shaded range of frequencies represents the frequencies in which a single-mode operation of the horns is guaranteed.

To study the behaviour of the designed FSS in terms of the angle of polarisation, the FSS has been simulated rotating it around its $\mathrm{Z}$-axis, from $\theta=0^{\circ}$ to $\theta=45^{\circ}$ as depicted in Fig. 9b. The simulated $\left|S_{21}\right|$ parameter for the different angles of polarisation is shown in Fig. 11. Although the minimum value of the $\left|S_{21}\right|$ parameter is modified with the angle of polarisation, the attenuation level remains over $10 \mathrm{~dB}$ in the non-shaded region. Consequently, the FSS presents an stable performance in terms of the angle of polarisation.

To study the behaviour of the designed FSS in terms of the angle of incidence, the FSS has been simulated rotating it around its Y-axis, from $\phi=0^{\circ}$ to $\phi=45^{\circ}$ as depicted in Fig. 9c. The simulated $\left|S_{21}\right|$ parameter for the different angles of incidence is shown in Fig. 12. The simulated FSS presents a stable performance in terms of its angle of incidence.

To study the behaviour of the designed FSS in terms of its radius of curvature, the FSS has been simulated bending it around a cylinder whose axis is parallel to the $\mathrm{X}$-axis, from a radius $R=400 \mathrm{~mm}$ to $R=600 \mathrm{~mm}$, as depicted in Fig. 9d. The minimum distance between the FSS and the transmiter horn is $D / 2$. The simulated $\left|S_{21}\right|$ parameter for the different radii of curvature is shown in Fig. 13. Although the minimum value of the $\left|S_{21}\right|$ parameter is modified with the radius of curvature, the attenuation level remains over $10 \mathrm{~dB}$ in the non-shaded region. Consequently, the FSS presents an stable performance in terms of the radius of curvature. 


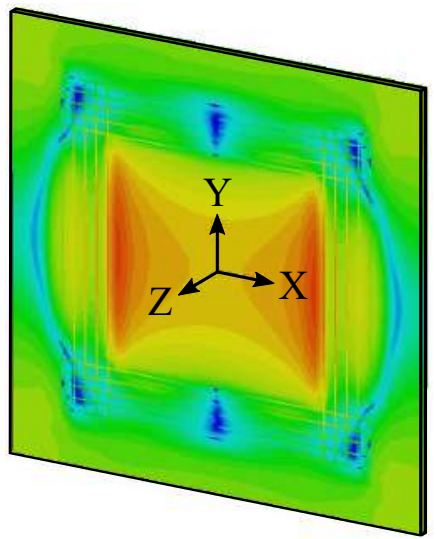

(a)

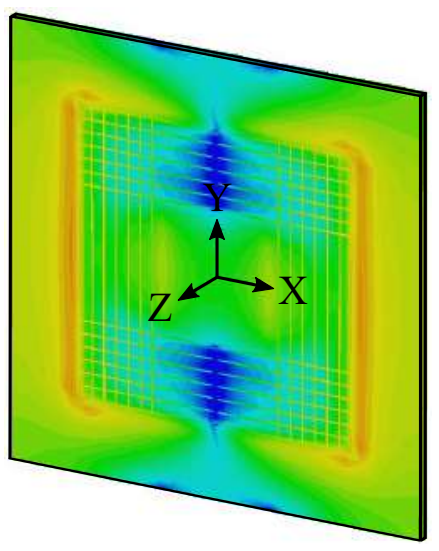

(c)

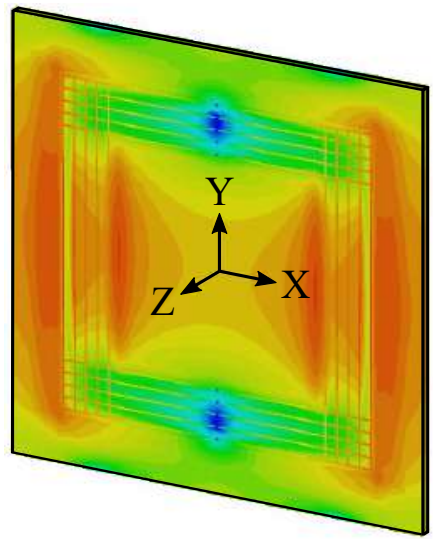

(b)

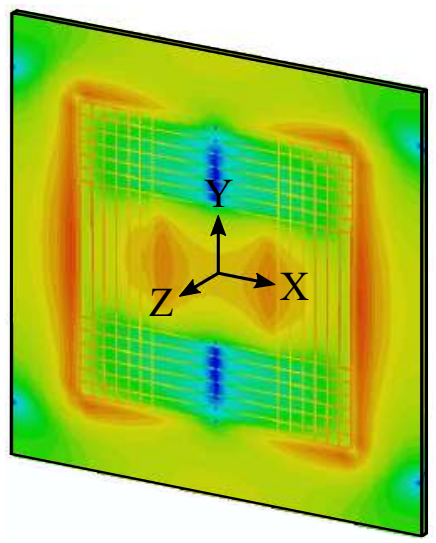

(d)

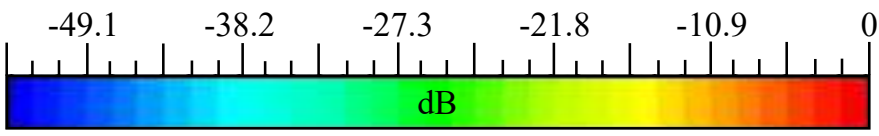

(e)

Fig. 8. Simulated individual behaviour of the normalised electric field (PBC). (a) Resonators in the top layer at $5.1 \mathrm{GHz}$. (b) Resonators in the top layer at $3.4 \mathrm{GHz}$. (c) Resonators in the bottom layer at $3.4 \mathrm{GHz}$. (d) Resonators in the bottom layer at $5.1 \mathrm{GHz}$. (e) Common scale for (a) to (d) representations

\section{FABRICATION PROCESS}

A finite $9 \times 9$ unit cells FSS has been manufactured using an industrial MüGrip loom. The manufacturing process has been based on satin weaving. The conductive warp yarns are previously mounted in the loom and the conductive weft threads are inserted in the fabric during the fabrication process leading to two conductive lattices in either side of the fabric, instead of the required square-rings. As the leftover conductive material has not been interwoven in the fabric during the manufacturing process, but it has been left loose, it then can be easily removed. A finishing process is needed after removing the fabric from the loom in order to achieve the requirements. Figure 14 depicts a generic finishing process which starts with the insertion of the fabric in liquid chemicals, then needling the fabric and finally applying heat to dry it. The manufactured FSS has been heated at $185^{\circ}$ on the frame of the stenter machine.

The top and bottom views of the manufactured prototype can be seen in Fig. 15a and b, whereas a magnification of

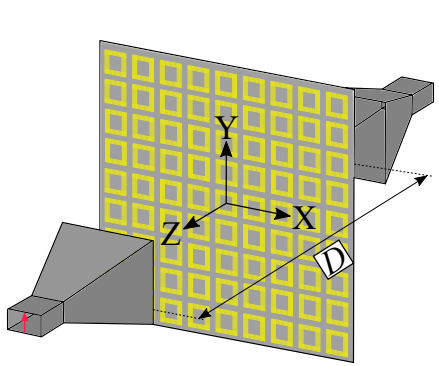

(a)

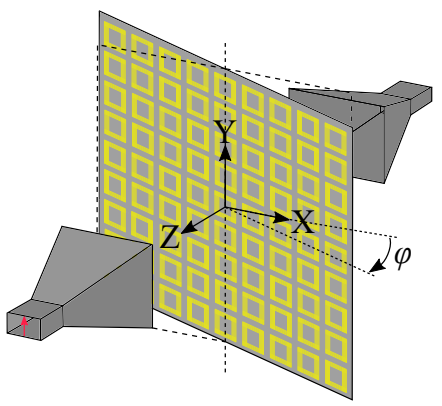

(c)

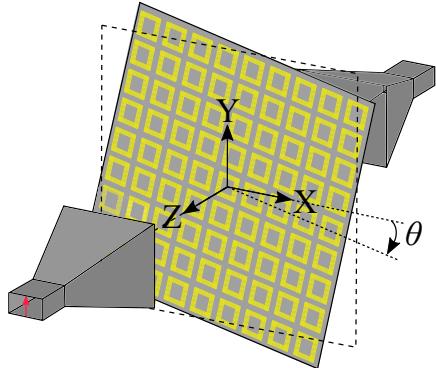

(b)

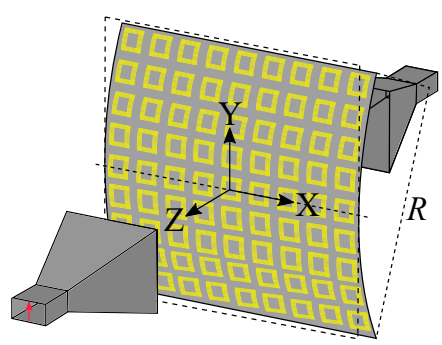

(d)
Fig. 9. Schematic drawings of the different set-ups to simulate the finite FSS $(D=1.5 \mathrm{~m})$. (a) Normal incidence. (b) Angle of polarisation. (c) Angle of incidence. (d) Radius of curvature.

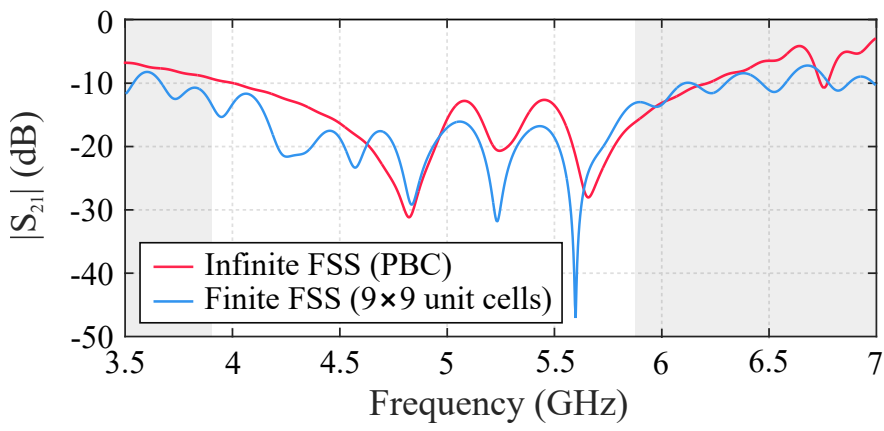

Fig. 10. Simulated $\left|S_{21}\right|$ parameter for an infinite FSS (unit cell and PBC) and for a finite FSS $(9 \times 9$ unit cells).

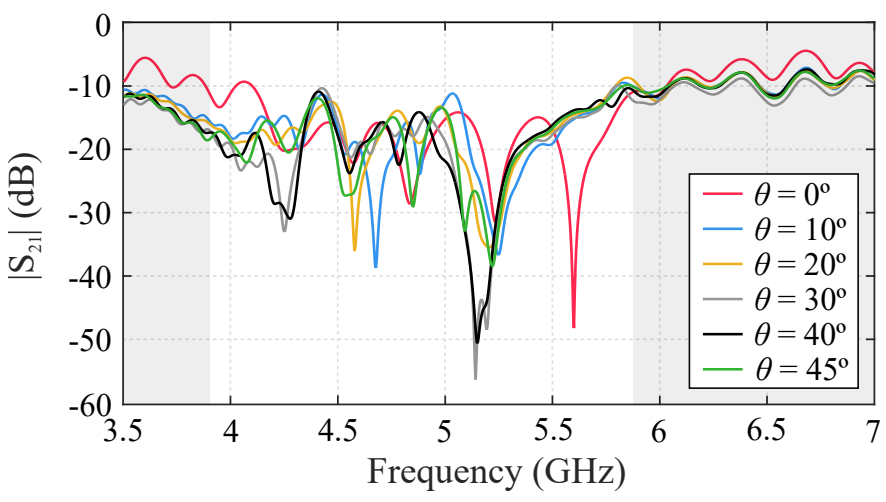

Fig. 11. Influence of the polarisation angle on the finite FSS performance. 


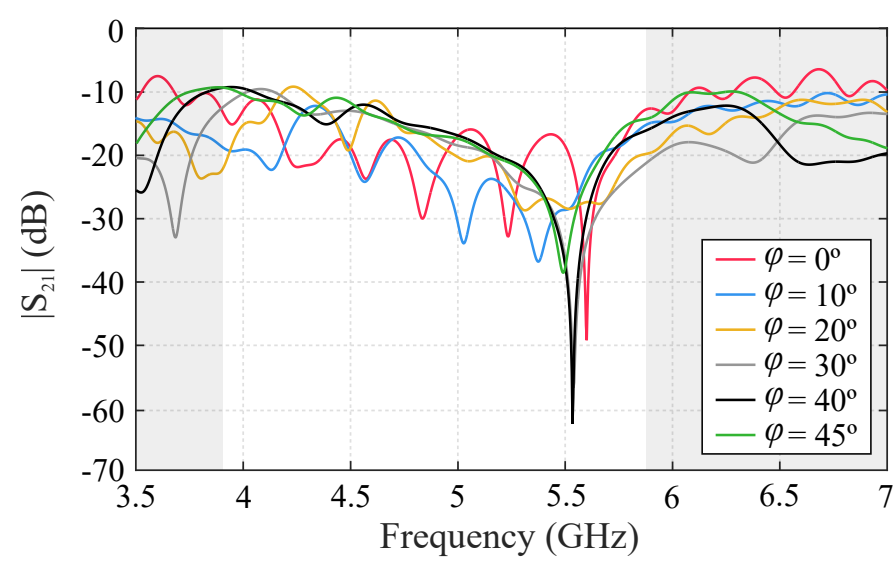

Fig. 12. Influence of the angle of incidence on the finite FSS performance.

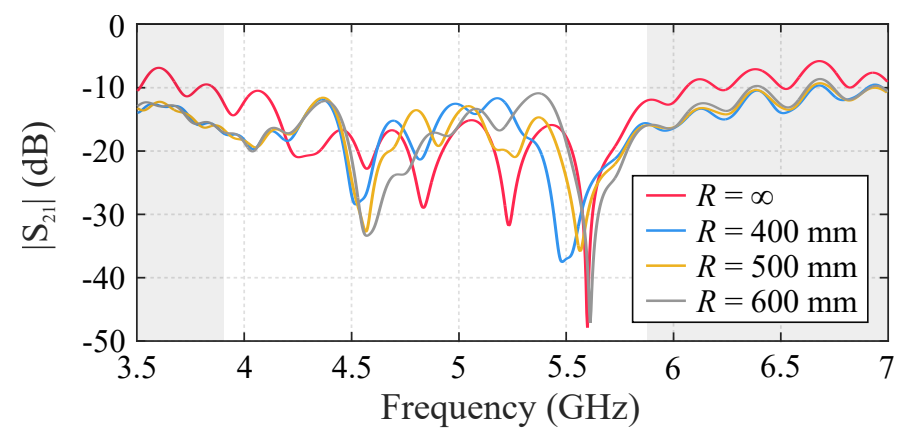

Fig. 13. Influence of the radius of curvature on the finite FSS performance.

the unit cells from the top and bottom layers are shown in Fig. $15 \mathrm{c}$ and d, respectively. A side view of the manufactured prototype is depicted in Fig. 15e.

\section{EXPERIMENTAL VALIDATION}

The prototype has been experimentally validated using the set-ups depicted in Fig. 16. The set-ups are composed of a wooden canvas, in which the prototype has been assembled and allows the required movements for the validation under different angles of incidence, two horn antennas mounted in rotary platforms for the validation of the different angles of polarisation and a vector network analyzer. The $S_{21}$ parameter between the horn antennas, for each set-up, calibrated by using the response between the antennas without the FSS, has been measured using an Agilent N5247A PNA-X vector network analyzer and compared with the simulations.

The FSS has been experimentally validated under normal incidence conditions using the set-up shown in Fig. 16a. The comparison between simulations and measurements can be

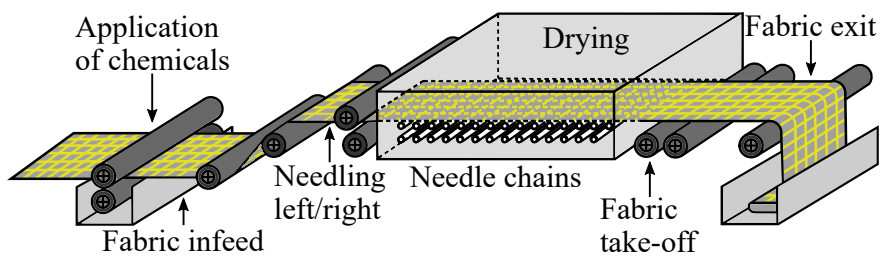

Fig. 14. Schematic drawing of the finishing process with a stenter machine.

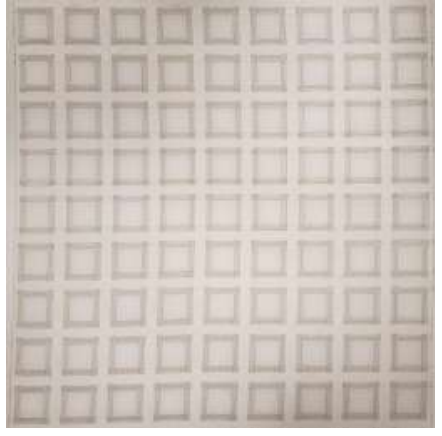

(a)

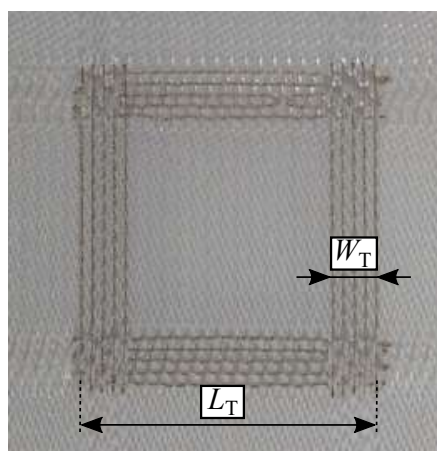

(c)

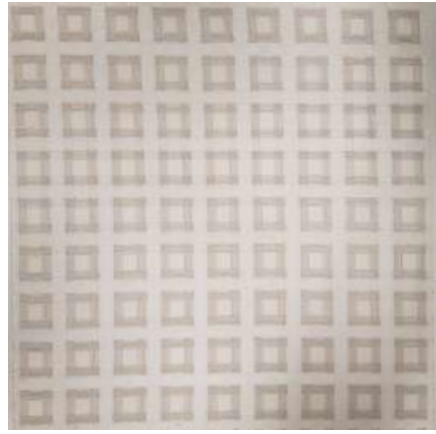

(b)

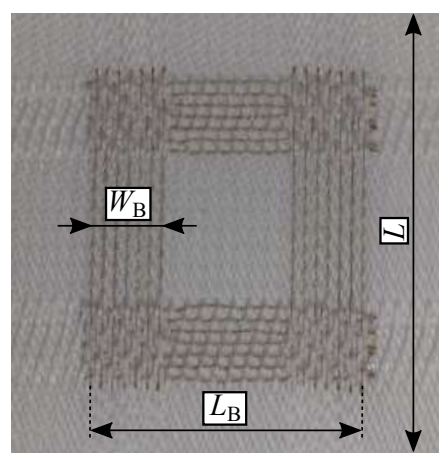

(d)

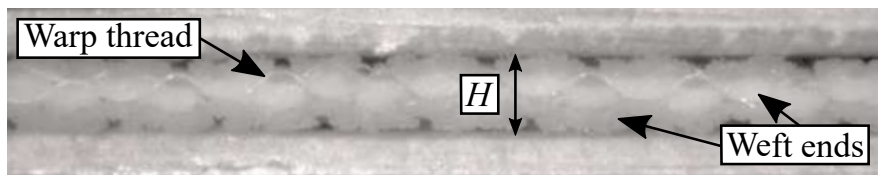

(e)

Fig. 15. Manufactured prototype. The dimensions are summarised in Table II. (a) Top view. (b) Bottom view. (c) Magnification of a unit cell from the top layer. (d) Magnification of a unit cell from the bottom layer. (e) Side view of the prototype compressed between two metallic sheets.

seen in Fig. 17. In the nominal frequency band of the horn antennas, represented as the non-shaded part of the figure, the manufactured FSS provides a bandstop behaviour, $\left|S_{21}\right|<-10$ $\mathrm{dB}$, which provides a $1.8 \mathrm{GHz}$ stopband centered at $5 \mathrm{GHz}$, with a $10 \mathrm{~dB}$ minimum attenuation. The predicted simulated data agree with the measurements, although the differences may be due to the approximations taken into account in the simulations. In the simulations, conductive flat strips are employed for the resonators, whereas the manufactured conductive woven paths are slightly longer due to the weaving curves. For this reason, the measured range of working frequencies is slightly lower than the simulated one.

The FSS has been experimentally validated for different angles of polarisation using the set-up shown in Fig. 16b in which the antennas have been mounted on the rotary platforms. The comparison between simulations and measurements is depicted in Fig. 18. In the single mode frequency range of the antennas, represented as the non-shaded part of the figure, the manufactured FSS has a bandstop behaviour. Consequently, the FSS exhibits a stable performance in terms of the polarisation angle. This represents an interesting point for the potential use of the proposed FSS as a wall cover to filter undesired frequencies. 


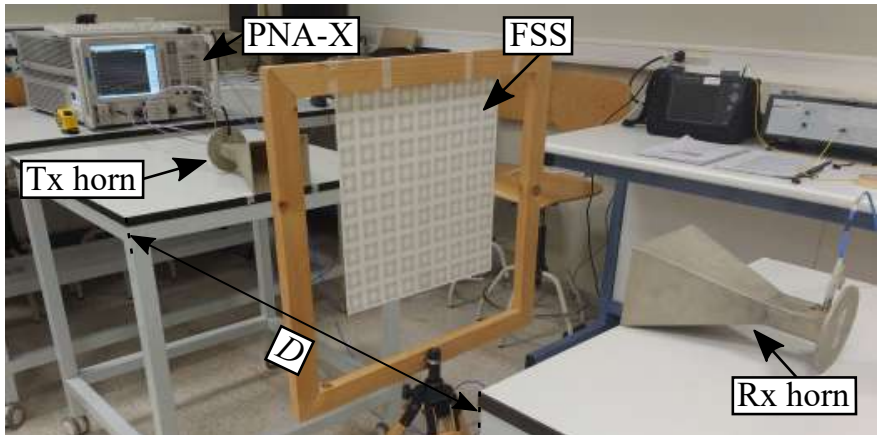

(a)

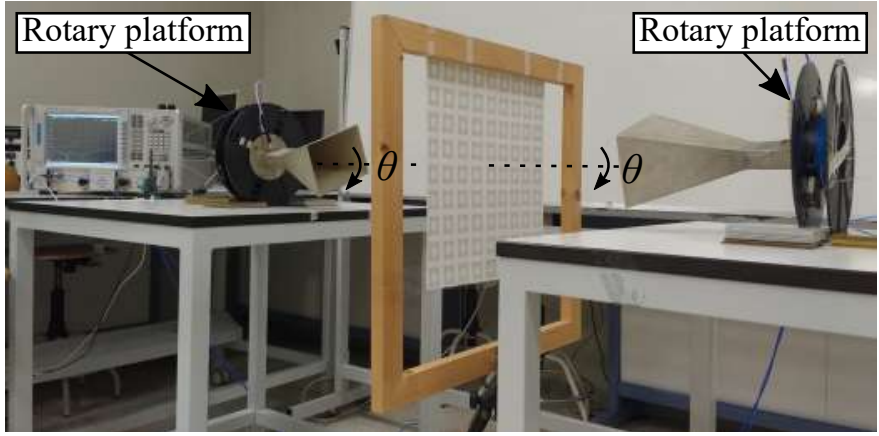

(b)

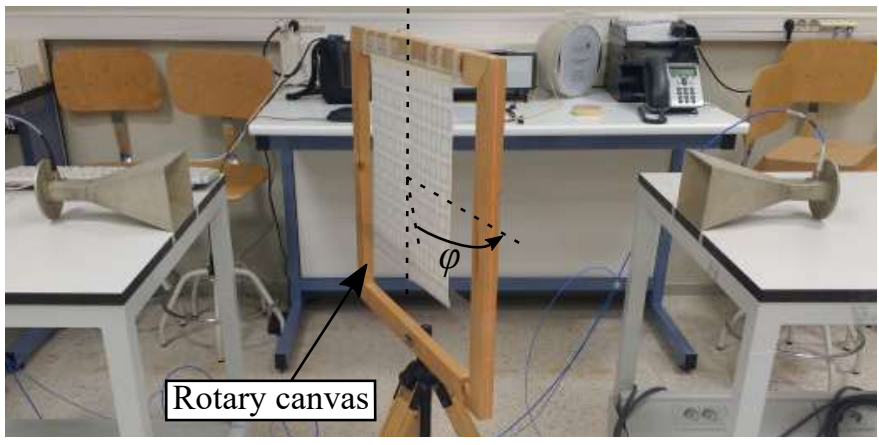

(c)

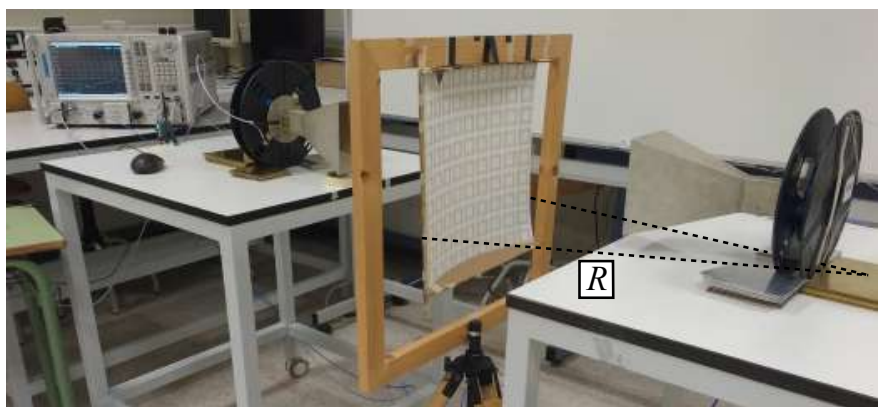

(d)

Fig. 16. Measurement set-ups $(D=1.5 \mathrm{~m})$. (a) Normal incidence. (b) Polarisation angle set-up. (c) Angle of incidence set-up. (d) Bending angle set-up.

The FSS has also been experimentally validated for different angles of incidence using the set-up shown in Fig. 16c with a rotary canvas. The comparison between simulations and measurements can be seen in Fig. 19. As predicted, in the single mode frequency range of the antennas, the manufactured FSS has a bandstop behaviour. Therefore, the FSS also exhibits

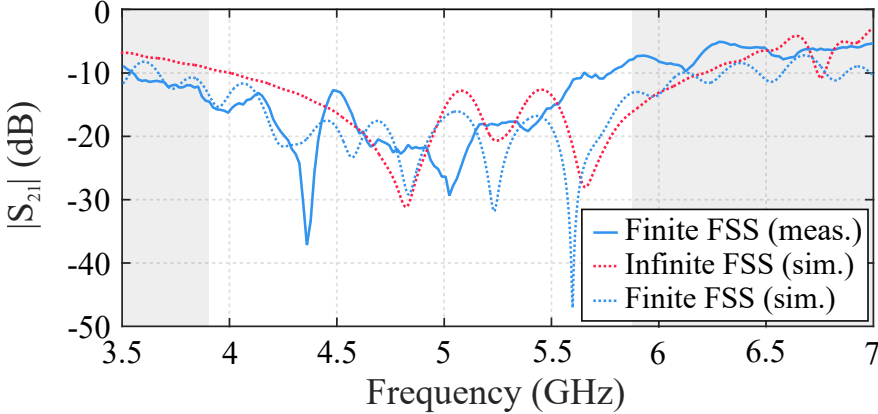

Fig. 17. Simulated $\left|S_{21}\right|$ parameter for an infinite FSS (unit cell and PBC) and for a finite FSS $(9 \times 9$ unit cells $)$ vs. measured $\left|S_{21}\right|$ parameter for the manufactured FSS

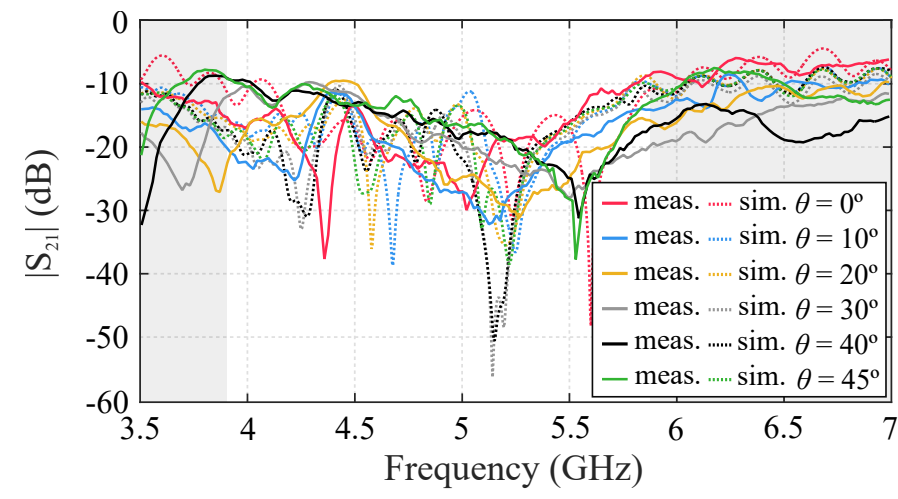

Fig. 18. Simulated and measured influence of the polarisation angle on the finite FSS performance.

a stable performance in terms of the angle of incidence, reinforcing its applicability in the aforementioned wall covering.

Due to its flexibility, the FSS has been experimentally validated for different radii of curvature using the set-up shown in Fig. 16d. The FSS has been bent around different cardboard pieces to achieve the required radius of curvature. The comparison between simulations and measurements is shown in Fig. 20. As predicted in the simulations, in the single mode frequency range of the antennas, the manufactured FSS has a bandstop behaviour. Consequently, the FSS exhibits a

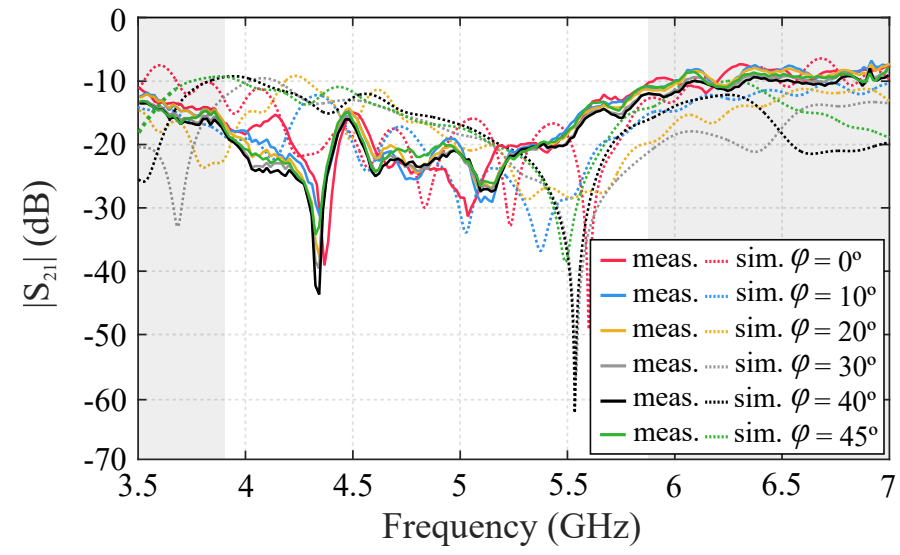

Fig. 19. Simulated and measured influence of the angle of incidence on the finite FSS performance. 


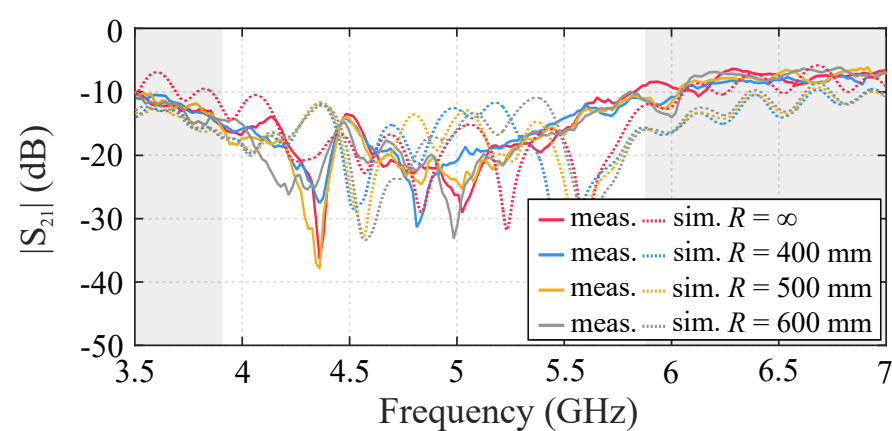

Fig. 20. Simulated and measured influence of the radius of curvature on the finite FSS performance.

stable performance in terms of the radius of curvature. This fact is the great interest for the before mentioned application regarding covering a wall. For this purpose, it is not necessary to attach the woven FSS to the wall, as it works properly under bent conditions. Consequently, the FSS can be used as a curtain, leading to an easily removable filter.

\section{CONCLUSION}

A novel broadband flexible fully textile-integrated bandstop FSS has been presented. To validate the design, a $9 \times 9$ unitcell prototype has been manufactured and experimentally characterised under different conditions, including different angles of polarisation, angles of incidence or radius of curvature. A good agreement between simulations and measurements has been achieved, experimentally verifying the theoretically predicted behaviour of the textile structure. Therefore, the manufactured FSS exhibits a stable performance in terms of the angle of polarisation, the angle of incidence and the radius of curvature.

The manufacturing procedure using industrial textile machinery provides the possibility of manufacturing flexible and large FSS, as opposed to other alternatives based in conventional substrates, solving the fabrication problems of large shielding surfaces.

\section{REFERENCES}

[1] B. A. Munk, Frequency selective surfaces: Theory and design. USA, Wiley-Interscience Publication, 2000, pp. 125.

[2] J. Tak and J. Choi, "A wearable metamaterial microwave absorber", in IEEE Antennas Wireless Propag. Lett., vol. 16, pp. 784-787, Aug., 2016.

[3] S. M. Rouzegar, A. Alighanbari and O. M. Ramahi, "Wideband uniplanar artificial magnetic conductors based on curved coupled microstrip line resonators," in IEEE Microw. Wirel. Compon. Lett., vol. 27, no. 4, pp. 326-328, Apr. 2017.

[4] W. Fu1 et al., "Polarization insensitive wide-angle tripleband metamaterial bandpass filter", presented in Progress in Electromagnetic Research Symposium (PIERS), Shanghai, China, Aug., 8-11, 2016.
[5] C. Xu et al., "A novel dual-stop-band FSS for infrared stealth applications", presented in Int. Applied Computational Electromagnetics Soc. Symp. (ACES), Suzhou, China, Aug., 1-4, 2017.

[6] M. Nauman and W. T. Khan, "A miniaturized dual-band stop frequency selective surface for $900 \mathrm{MHz}$ and 1800 MHz bands shielding", presented in 11th European Conf. on Antennas and Propag. (EUCAP), Paris, France, Mar., 19-24, 2017.

[7] X. Xiong et al., "WiFi band-stop FSS for increased privacy protection in smart building", presented in IEEE 6th Int. Symp. on Microw. Antenna Propag. and EMC Technol. (MAPE), Shanghai, China, Oct., 28-30, 2015, pp. 826828.

[8] N. Liu et al., "A Design Method for Synthesizing Wideband Band-Stop FSS via Its Equivalent Circuit Model", in IEEE Antennas and Wireless Propag. Lett., vol. 16, pp. 2721-2725, Aug., 2017.

[9] M. Yan et al., "A novel miniaturized dual-stop-band FSS for Wi-Fi application", presented in Progress in Electromagnetic Research Symp. (PIERS), Shanghai, China, Aug., 8-11, 2016.

[10] M. H. Nisanci et al., "Experimental validation of a 3D FSS designed by periodic conductive fibers part-2: bandstop filter characteristic", in IEEE Trans. on Electromagnetic Compatibility, vol. 59, no. 6, pp. 1835-1840, Jun. 2017.

[11] L. Li et al., "All-dielectric metamaterial band stop frequency selective surface via high-permittivity ceramics", presented in Progress in Electromagnetic Research Symp. (PIERS), Shanghai, China, Aug., 8-11, 2016.

[12] W. Fu et al., "Polarization insensitive wide-angle tripleband metamaterial bandpass filter", in Journal of Physics D: Applied Physics, vol. 49, no. 28, 2016.

[13] M. Fallah, A. Ghayekhloo and A. Abdolali, "Design of frequency selective band stop shield using analytical method", in Journal of Microw., Optoelectronics and Electromagnetic Applications, vol. 14, no. 2, Dec. 2015.

[14] M. M. Tahseen and A. A. Kishk, "Flexible and portable textile-reflectarray backed by frequency selective surface", in IEEE Antennas Wireless Propag. Lett., vol. 17, no. 1, pp. 46-49, Jan. 2018.

[15] W. G. Whittow et al., "Printed frequency selective surfaces on textiles", in Electr. Lett., vol. 50, no. 13, pp. 916-917, June 192014.

[16] M. Ghebrebrhan et al., "Textile frequency selective surface", in IEEE Microw. Wireless Components Lett., vol. 27, no. 11, pp. 989-991, Nov. 2017.

[17] L. Alonso-González et al. "Novel parametric electromagnetic modelling to simulate Textile Integrated Circuits," presented at the Int. Conf. Numerical Electromagnetic and Multiphysics Modeling and Optimization for RF, Microwave, and Terahertz Applications (NEMO), Seville, Spain, May 17-19, 2017.

[18] L. Alonso-González et al., "On the techniques to develop millimeter-wave textile integrated waveguides using rigid warp threads", in IEEE Trans. Microw. Theory and Techn., vol. PP, no. 99, pp. 1-11. 
[19] Shieldex Trading, "Shieldex ${ }^{\circledR}$ Conductive Twisted Yarn Silver Plated Nylon 66 Yarn 117/17 dtex 2ply," PN\# 260121011717, 2010 [Revised Jan. 2012]. [Online]. Available: www.shopvtechtextiles.com/assets/ images/260121011717.pdf. [Accessed Jan. 21, 2018].

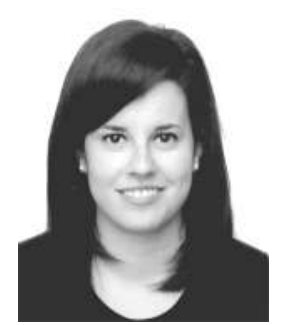

Leticia Alonso-González (S'14) received the M.Sc. degree in telecommunications engineering from the University of Oviedo, Gijón, Spain, and the M.Sc. degree in Systems and Control Engineering from the National University of Distance Learning (UNED), Spain, in 2014 and 2018, respectively, and she is currently working toward the Ph.D. degree at the University of Oviedo.

Since 2014, she has been working as a researcher in the Signal Theory and Communications Group, University of Oviedo. She was a Visiting Scholar at the George Green Institute for Electromagnetics Research at the University of Nottingham (UK) in 2017. Her main research effort is focused on the design, simulation and manufacturing techniques to develop microwave and millimetre-wave passive circuits and antennas fully integrated in textile technology.

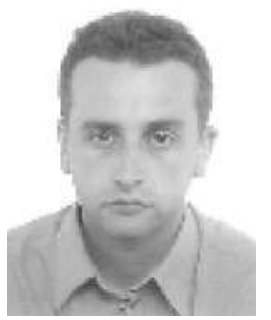

Samuel Ver-Hoeye (M'05) received the M.Sc. degree in electronic engineering from the University of Gent, Gent, Belgium, in 1999, and the Ph.D. degree from the University of Cantabria, Santander, Spain, in 2002 .

$\mathrm{He}$ is currently an Associate Professor with the Department of Electrical and Electronic Engineering, University of Oviedo, Gijón, Spain. His main research interests are focused on the design and analysis of nonlinear oscillator based circuits, millimeter wave and terahertz antennas, circuits and systems, graphene based components, and textile integrated circuits and antennas.

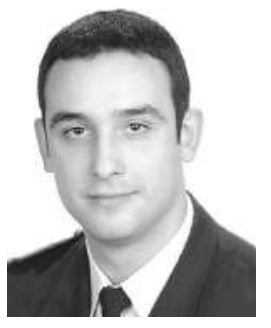

Miguel Fernández-García received the M.Sc. degree in telecommunication engineering, the M.Sc. degree in information technology and mobile communications and the Ph.D. degree from the University of Oviedo, Gijón, Spain, in 2006, 2010, and 2010, respectively.

From 2006 to 2008, he was a Research Fellow with the Signal Theory and Communications Group, University of Oviedo, where he has been an Associate Professor since 2008. His current research interests include nonlinear analysis and optimization techniques for the design of oscillator-based circuits, active antennas and frequency multipliers and mixers at the microwave, millimeter/submillimeterwave, and terahertz frequency bands.

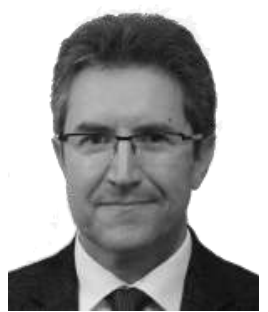

Fernando Las-Heras Andrés (M'86-SM'08) received the M.S. and Ph.D. degrees in telecommunication engineering from the Technical University of Madrid (UPM), Madrid, Spain, in 1987 and 1990, respectively.

He was a National Graduate Research Fellow from 1988 to 1990 and an Associate Professor from 1991 to 2000 with the Department of Signal, Systems and Radiocom, UPM. He was the Vice-Dean of

Telecommunication Engineering with the Technical School of Engineering, Gijón, from 2004 to 2008 He was a Visiting Researcher with Syracuse University, New York, NY, USA, and a Visiting Lecturer with the National University of Engineering, Lima, Peru, and the École Supérieure d'Ingénieurs en Génie Électrique, Rouen, France. He has been the Head with the Signal Theory and Communications Research Group, Department of Electrical Engineering, University of Oviedo, Gijón, Spain, since 2001, and has been a Full Professor since 2003. He has authored over 450 technical journal and conference papers in the areas of electromagnetic radiation, propagation and scattering theory and applications as well as inverse problems.

Dr. Las-Heras held the Telefónica Chair on "RF Technologies, ICTs Applied to Environment and Climate Change", and ICTs and Smartcities," from 2005 to 2015. He was a member of the Board of Directors of the IEEE Spain Section from 2012 to 2015, and a member of the Science, Technology and Innovation Council of Asturias, Spain, in 2010. He is a member of the IEEE Microwaves and Antennas Propagation Chapter (AP03/MTT17) Board from 2016 to 2018 